\title{
Novel respiratory viruses: what should the clinician be alert for?
}

\author{
Authors: Brendan Payne ${ }^{A}$ and Richard Bellamy ${ }^{B}$
}

\begin{abstract}
Since 1990, several novel respiratory viruses affecting humans have been described. In this review, we focus on three pathogens that have caused significant human mortality and raise important public health concerns: severe acute respiratory syndrome (SARS)-coronavirus, Middle East respiratory syndrome (MERS)-coronavirus and avian influenza A viruses (H5N1 and H7N9). Novel respiratory viruses have the potential to instil fear in the public and physicians alike if they are associated with a high case fatality rate. Those viruses with a significant potential for onward human-to-human transmission (including in healthcare settings) might present significant challenges for national public health services and local hospital infection control.
\end{abstract}

KEYWORDS: SARS-CoV, MERS-CoV, avian influenza, H5N1, H7N9, coronavirus

\section{Introduction}

In this review, we focus on the approach to the investigation and management of novel respiratory viruses relevant to the receiving clinician in a UK healthcare setting. By its nature, national guidance for the management of novel viruses, and the risk assessments on which it is based, are liable to change. Therefore, useful online resources are given in Box 1 .

\section{Novel coronaviruses}

Coronaviruses $(\mathrm{CoV})$ are so named for their 'crown' of surface spikes visible under electron microscopy. Historically, CoV have received relatively little attention from the medical community. Two coronaviruses (CoV-229E and CoV-OC43) were identified as major causes of the common cold during the 1960s. The emergence of severe acute respiratory syndrome (SARS) reignited interest in human $\mathrm{CoV}$ infection. A second human $\mathrm{CoV}$ leading to severe illness, Middle East respiratory syndrome (MERS) CoV, emerged in 2012.

Multiple $\mathrm{CoV}$ are found in bats and it is likely that bats were the original reservoir for $\mathrm{CoV}$ in terrestrial mammals, including

Authors: ${ }^{A}$ academic clinical lecturer, Centre for Clinical Infection, James Cook University Hospital, UK and Newcastle University, Newcastle-upon-Tyne, UK; ${ }^{B}$ consultant physician, Newcastle University, Newcastle-upon-Tyne, UK. humans. ${ }^{1}$ However, many CoV are readily transmissible between different terrestrial mammalian species; for example, human CoV-OC43 is almost identical to bovine $\mathrm{CoV}$. CoV have large RNA genomes that readily undergo recombination. ${ }^{2}$ These features make $\mathrm{CoV}$ prime candidates for interspecies transmissions and, therefore, novel human infections.

\section{SARS coronavirus}

SARS-CoV emerged in Guangdong, China, during late 2002. Ultimately, 8,273 cases were detected in 37 countries throughout the world. In total, 775 deaths were recorded (a case fatality rate of 9.6\%). No further cases have been reported since July 2003. Horseshoe bats are believed to be the natural reservoir, and have asymptomatic infection. ${ }^{3}$ From this reservoir, infection spread to palm civet cats sold as exotics in 'wet markets' in Guangdong and thence to humans. ${ }^{4}$ However, most human cases were the result of subsequent human-tohuman transmissions.

Clinically, SARS was characterised by severe pneumonia or acute respiratory distress syndrome (ARDS), as well as diarrhoea in a significant minority of cases. The epidemic was characterised by many large hospital-based outbreaks with transmissions to healthcare workers (HCWs). Recirculation in hospital air-conditioning systems is thought to have had a role in at least some nosocomial outbreaks. It is thought that patients were most infectious during the latter stages of their illness, and from the lower rather than the upper respiratory tract. ${ }^{5}$ These factors presumably account for relatively higher numbers of transmissions in hospitals compared with the community.

Many treatments were tried for SARS, including: convalescent serum, intravenous immunoglobulin, ribavirin, interferon- $\alpha$

\section{Box 1. Useful resources}

Public Health England (PHE; formerly the Health Protection Agency (HPA)): www.gov.uk/government/organisations/publichealth-england

World Health Organisation (WHO): www.who.int

ProMED (International Society for Infectious Diseases): www.promedmail.org. The website provides daily updates on emerging infections. 
and HIV protease inhibitors. No approach showed evidence of benefit, and some showed possible harm (corticosteroids and ribavirin). ${ }^{6}$

\section{What is the relevance of SARS-CoV for current UK practice?}

The likelihood of SARS-CoV re-emerging in humans remains uncertain. It is presumed that the virus still exists in the natural reservoir; however, it has not re-emerged over a period of 10 years. It is perhaps more likely that another novel $\mathrm{CoV}$ might emerge in humans in the future. The world is currently defined by the World Health Organisation (WHO) as being in an 'interepidemic' period. To remain vigilant to the possibility of SARS-CoV re-emergence, Public Health England (PHE) and the WHO retain definitions for possible cases (Table 1).

\section{MERS coronavirus}

In September 2012, an Egyptian virologist working in Saudi Arabia isolated a novel CoV from a fatal case of ARDS and renal failure. ${ }^{7}$ The majority of subsequent cases have also originated in Saudi Arabia. Essentially all countries in the Middle East are considered (by PHE) as potentially at risk for MERS-CoV. In addition, there have been cases imported into many countries in Europe and North America, including the UK. As of April 2014, there had been 345 reported cases and 107 deaths (a 31\% case fatality rate). There was a large spike in reported cases in April 2014.

Much remains to be determined about the natural reservoir and modes of transmission of MERS-CoV. As with SARS$\mathrm{CoV}$, it is likely that the virus originates from a bat $\mathrm{CoV}$. The best candidate for the natural reservoir is the dromedary camel. A high proportion of camels show serological evidence of previous infection, and a small percentage of apparently healthy camels at an abattoir showed the presence of current infection (viral RNA detected). ${ }^{8,9}$ To date, there has been one documented case of direct transmission from camel to human; ${ }^{10}$ however, if camels are the source of human infections, it seems likely that most human cases are the result of indirect transmission, the route of which remains unclear. As with SARS-CoV, there have now been several outbreaks involving human-to-human spread within healthcare facilities. ${ }^{11}$

Clinically, most cases of MERS-CoV have presented with severe pneumonia and/or ARDS, although there have been cases with minimal symptoms documented. A few patients have presented with diarrhoea, and renal failure is sometimes seen.

\section{What is the pandemic potential of MERS-CoV?}

Currently, human-to-human transmission of MERS-CoV appears limited, particularly outside a hospital setting. It is reassuring that, so far, contacts of imported cases have not established sustained transmissions. The SARS-CoV epidemic 'burnt out' for reasons that are not entirely clear. By contrast, the mildly pathogenic CoV-OC43 and 229E became globally established in humans at some point in the past, presumably originally from a zoonotic source.

\section{Avian influenza}

\section{What do we mean by 'avian influenza'?}

Influenza A viruses are classified based on their $\mathrm{H}$ (haemagglutinin) and $\mathrm{N}$ (neuraminidase) surface proteins. To date, only viruses of H1-H3 and N1-N2 types have caused epidemic influenza in humans. By contrast, viruses of H1H16 and N1-N9 occur in wild birds. In temperate regions, influenza occurs as seasonal epidemics. Small differences (mutations) in circulating virus from year to year (genetic drift), allow re-infection of previously exposed persons, despite the influenza A type remaining unchanged. However, influenza A pandemics result from genetic reassortment (genetic shift) of the multiple segments of RNA within the virus. Such reassortment is facilitated where humans live in close proximity to domestic poultry and other animals, such as swine. The most recent pandemic was the result of the influenza A (H1N1pdm2009) strain, which was a novel reassortant virus containing multiple genes from different swine lineage influenza A viruses. ${ }^{12}$

By contrast, the term 'avian influenza' (as applied to a human infection) means infection of a human with a virus that is actively circulating in birds without further genetic reassortment. Several avian influenza A virus types have caused occasional human infections, which are often mild, such as conjunctivitis (H7N7) (13). However, two avian influenza A viruses have caused severe human disease in recent years: H5N1 and H7N9. Furthermore, three cases of fatal infection with avian influenza A (H10N8) have very recently been described. ${ }^{14}$

\section{Avian influenza A (H5N1)}

Influenza A (H5N1) first emerged during 1997, and then reemerged in 2003 (at a similar time to SARS-CoV). Cases have centred principally on China, although the distribution of the virus in bird populations is wide and human cases have now been reported in 15 countries, scattered across Asia and Africa. From 2003 until March 2014, 664 laboratory-confirmed human cases had been reported, with 391 deaths (a 59\% case fatality rate). ${ }^{15}$ Almost all cases were the result of bird-to-human transmission, but given the scale of outbreaks in birds, it appears that bird-to-human transmission cannot occur readily. Human-to-human transmissions are documented but appear to be rare thus far. As a consequence, the main control measures have centred on good surveillance of avian influenza in humans and bird populations and, where necessary, culls and closure of 'wet markets'. Such surveillance in China was responsible for the prompt recognition of recent human cases of $\mathrm{H} 7 \mathrm{~N} 9$ and H10N8.

\section{Avian influenza A (H7N9)}

Avian influenza A (H7N9) emerged in humans during early 2013. Thus far, cases have been essentially confined to China, with the exception of one imported case in Malaysia. To date, there have been two waves: in spring 2013 and a larger wave from December 2013. In total, 375 cases had been reported by February 2014, with 115 deaths (a 31\% case fatality rate). Most cases were reported in middle-aged and older men. Most cases were associated with severe respiratory illness, although some milder cases have been reported in children. ${ }^{16}$ 


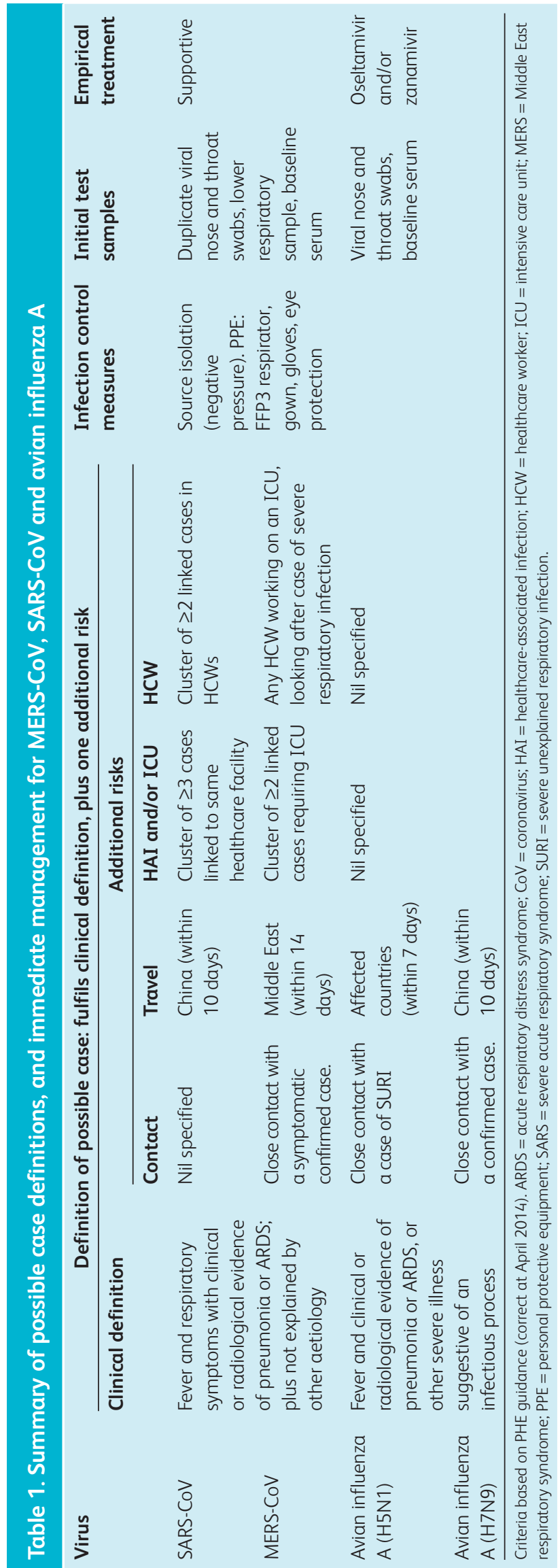


What is the pandemic potential of avian influenza viruses?

Before the emergence of the novel influenza A (H1N1pdm2009), $\mathrm{H} 5 \mathrm{~N} 1$ had been considered as the most likely candidate for the next pandemic. Currently, the determinants of pandemic potential in avian influenza viruses are relatively poorly understood. On the one hand, it is perhaps reassuring that H5N1 has not become adapted to human-to-human spread despite circulating in birds and causing sporadic human cases for over 10 years. On the other hand, recent work shows that a limited number of passages of the virus in a ferret model can generate a mammalian-adapted virus. ${ }^{17}$ A growing list of molecular determinants of host adaptation are being defined and, in the future, this will aid surveillance of avian influenza viruses. ${ }^{18}$

A 'pre-pandemic' influenza A (H5N1) vaccine has been developed, although it is uncertain whether this would offer protection against a mutated pandemic strain. A new influenza vaccine takes approximately 6 months to develop and, thus, is not an available option during the first wave of a pandemic. Many countries stockpile oseltamivir for use in the event of a pandemic, although this approach is not without controversy. ${ }^{19}$

\section{When should a novel respiratory virus be suspected clinically?}

In Table 1, we summarise the current (UK PHE, at April 2014) guidance on the possible case definitions and initial management (infection control, testing and treatment) for suspected cases of MERS, SARS and avian influenza. From the table, the reader will note some common themes. First, the clinician needs to be alert to cases of acute respiratory infection, particularly if severe.

$>$ Does the patient have fever (or history of fever), cough and evidence of lung consolidation (clinical or radiological evidence, including of ARDS)?

If so, further information should then be sought, following this general approach.

$>$ Is there a travel history (last 7-14 days)?

$>$ Are there other relevant contacts (poultry or other animal)?

$>$ Is there a history of contact with a known case of a specific respiratory virus, or a case of severe unexplained respiratory illness (SURI)?

$>$ Does the patient work as an HCW (particularly in an intensive care unit (ICU) setting)?

As can be seen from Table 1, the possible case definitions vary between viruses, and can also change with evolution of risk assessments (WHO or PHE). If the clinician suspects a case on clinical grounds, they should always check the additional criteria online (links listed in Box 1), and/or take further advice from their local microbiologist, virologist or infectious diseases physician.

For example, for suspected MERS-CoV infection, if an HCW who works on an ICU caring for patients with acute severe respiratory illness, presents with fever and SURI, this fulfils the possible case definition, irrespective of travel history. Similarly, a cluster of two or more linked cases of patients with fever and SURI requiring ICU admission would also fulfil the possible case definition, irrespective of travel history. Importantly for the UK-based clinician, these criteria remind us that if humanto-human transmission of a novel respiratory virus were to become established in the UK (perhaps following transmission from a mildly symptomatic imported case), then we need to be mindful of the possibility of cases presenting without a travel history.

\section{How should a possible case of a novel respiratory virus be managed by the admitting clinician?}

On suspicion of a possible case of a novel respiratory virus infection, the following steps should be followed:

> isolate the patient (side room, ideally with negative pressure ventilation)

> institute appropriate personal protective equipment: gown, gloves, eye protection and FFP3 respirator (surgical face masks do not give adequate protection against infectious aerosols)

$>$ inform local microbiologist

> clinician or local microbiologist should inform the regional PHE laboratory (duty virologist), and the local PHE health protection team

$>$ take appropriate diagnostic samples as advised.

In a suspected case of MERS-CoV, full diagnostic samples comprise duplicate viral nose and throat swabs plus a lower respiratory sample (because some cases of MERS-CoV have only been detected in sputum) and a baseline serum sample. The regional PHE laboratory will coordinate the testing of one nose and throat sample at their laboratory for a respiratory viral multiplex PCR, and the transport of the other samples to the national MERS-CoV testing laboratory.

In a suspected case of avian influenza A, viral nose and throat swabs and baseline serum are required. Swabs will be urgently tested locally for a generic influenza A PCR target, and targets specific to circulating seasonal influenza A viruses. If the results of these tests show that the combination of influenza $\mathrm{A}$ is detected, but untypable, the sample will be referred urgently for specific avian influenza testing.

In suspected avian influenza, empirical oseltamivir should be started pending the results of viral testing. There is no established treatment for MERS-CoV. PHE and the International Severe Acute Respiratory \& Emerging Infection Consortium (ISARIC) have produced a clinical decision support tool, based largely on the limited data for SARS-CoV.

For emerging infections such as MERS-CoV, the public health management approach will be based on the first few hundred ('FF100') model. This presents a standardised form of detailed clinical, epidemiological and virological monitoring, which will inform the management of later cases and allow the determination of basic epidemiological parameters, such as the rate of secondary transmissions.

\section{Conclusions}

Recent novel respiratory viruses causing severe human illness remind us of the very real threat that such infections pose to public health. Recent advances in epidemiological and molecular surveillance techniques have made some improvements in our ability to manage the public health 
response to such viruses, but many aspects remain unknown. Physicians should always refer to the latest guidance in the event of managing a suspected case. Although novel respiratory viruses are perhaps less likely to first arise in the UK, the extent and rapidity of international travel to and from the UK means that early cases of a novel infection might be seen in this country. Therefore, vigilance by admitting physicians for small clusters (two or three cases) of SURI, particularly in an ICU setting and/or where HCWs are affected is a key early-warning system.

\section{References}

1 Vijaykrishna D, Smith GJ, Zhang JX et al. Evolutionary insights into the ecology of coronaviruses. J Virol 2007;81:4012-20.

2 Lai MM, Cavanagh D. The molecular biology of coronaviruses. Adv Virus Res 1997;48:1-100.

3 Lau SK, Woo PC, Li KS et al. Severe acute respiratory syndrome coronavirus-like virus in Chinese horseshoe bats. Proc Natl Acad Sci USA 2005;102:14040-5.

4 Guan Y, Zheng BJ, He YQ et al. Isolation and characterization of viruses related to the SARS coronavirus from animals in southern China. Science 2003;302:276-8.

5 Peiris JS, Chu CM, Cheng VC et al. Clinical progression and viral load in a community outbreak of coronavirus-associated SARS pneumonia: a prospective study. Lancet 2003;361:1767-72.

6 Stockman LJ, Bellamy R, Garner P. SARS: systematic review of treatment effects. PLoS Med 2006;3:e343.

7 Zaki AM, van Boheemen S, Bestebroer TM, Osterhaus AD, Fouchier RA. Isolation of a novel coronavirus from a man with pneumonia in Saudi Arabia. N Engl J Med 2012;367:1814-20.

8 Reusken CB, Haagmans BL, Muller MA et al. Middle East respiratory syndrome coronavirus neutralising serum antibodies in dromedary camels: a comparative serological study. Lancet Infect Dis 2013;13:859-66.
9 Chu DK, Poon LL, Gomaa MM et al. MERS coronaviruses in dromedary camels, Egypt. Emerg Infect Dis 2014;20:1049-53.

10 Memish ZA, Cotten M, Meyer B et al. Human infection with MERS coronavirus after exposure to infected camels, Saudi Arabia, 2013. Emerg Infect Dis 2014;20:1012-5.

11 Memish ZA, Zumla AI, Assiri A. Middle East respiratory syndrome coronavirus infections in health care workers. $N$ Engl J Med 2013;369:884-6.

12 Smith GJ, Vijaykrishna D, Bahl J et al. Origins and evolutionary genomics of the 2009 swine-origin H1N1 influenza A epidemic. Nature 2009;459:1122-5.

13 Koopmans M, Wilbrink B, Conyn M et al. Transmission of H7N7 avian influenza $\mathrm{A}$ virus to human beings during a large outbreak in commercial poultry farms in the Netherlands. Lancet 2004;363:587-93.

14 Chen H, Yuan H, Gao R et al. Clinical and epidemiological characteristics of a fatal case of avian influenza A H10N8 virus infection: a descriptive study. Lancet 2014;383:714-21.

15 Beigel JH, Farrar J, Han AM et al. Avian influenza A (H5N1) infection in humans. N Engl J Med 2005;353:1374-85.

16 Gao HN, Lu HZ, Cao B et al. Clinical findings in 111 cases of influenza A (H7N9) virus infection. N Engl J Med 2013;368:2277-85.

17 Herfst S, Schrauwen EJ, Linster M et al. Airborne transmission of influenza A/H5N1 virus between ferrets. Science 2012;336:1534-41.

18 Schrauwen EJ, Fouchier RA. Host adaptation and transmission of influenza A viruses in mammals. Emerg Microbes Infect 2014;3:e9.

19 Jefferson T, Jones M, Doshi P et al. Oseltamivir for influenza in adults and children: systematic review of clinical study reports and summary of regulatory comments. BMJ 2014;348:g2545.

Address for correspondence: Dr B Payne, Institute of Genetic Medicine, Newcastle University, International Centre for Life, Central Parkway, Newcastle-upon-Tyne, NE1 3BZ, UK.

Email: brendan.payne@newcastle.ac.uk 